\title{
Zur Zitierweise
}

Fontanes Werke werden im allgemeinen im laufenden Text nach der UllsteinTaschenbuchausgabe zitiert. Dabei gelten folgende Siglen:

ATO = Aus den Tagen der Okkupation

$\mathrm{C}=$ Cécile

CFS = Friedrich Christian Scherenberg und das literarische Berlin von 1840

bis 1860

DK $=$ Der Deutsche Krieg

$\mathrm{DP}=$ Die Poggenpuhls

$\mathrm{DS}=$ Der Stechlin

$\mathrm{EB}=$ Effi Briest

FJT $=$ Frau Jenny Treibel

GP $=$ Graf Petöfy

IW = Irrungen, Wirrungen

$\mathrm{K}=$ Kriegsgefangen

$\mathrm{LA}=$ L'Adultera

$\mathrm{MK}=$ Meine Kinderjahre

$\mathrm{MM}=$ Mathilde Möhring

$\mathrm{Q}=$ Quitt

$\mathrm{S}=$ Stine

SHK = Der Schleswig-Holsteinsche Krieg

SvW = Schach von Wuthenow

VdS 1-4 = Vor dem Sturm, 4 Bde.

VVNR $=$ Von, vor und nach der Reise

W 1-5 = Wanderungen durch die Mark Brandenburg, 5 Bde.

$\mathrm{ZD}=$ Von Zwanzig bis Dreißig

In Aufsätzen, die sich mit einem bestimmten Roman beschäftigen, wird bei diesem Roman die jeweilige Sigle weggelassen und nur die Seitenzahl gegeben.

Bei den übrigen, in den Anmerkungen belegten Werken Fontanes werden folgende Siglen verwendet:

$\mathrm{AFA}=$ Fontane-Ausgabe des Aufbau Verlags; dabei ist:

AFG 1-3 = Gedichte, 3 Bde.

AFR 1-8 = Romane, 8 Bde.

$\mathrm{AFS}=$ Autobiographische Schriften, 3 Bde. 
DuD 1-2 = Theodor Fontane. Der Dichter über sein Werk, hg. von Richard Brinkmann in Zusammenarbeit mit Waltraud Wiethölter, 2 Bde. München 1977 (= dtv 6073/6074).

HB 1-4 = Hanser Fontane-Ausgabe, Abteilung IV. Briefe, 4 Bde.

NFA = Nymphenburger Fontane-Ausgabe.

Wo weitere Fontane-Ausgaben benutzt wurden, sind diese in den Anmerkungen spezifiziert.

Weiterhin werden folgende Werke abgekürzt zitiert:

Briefe Friedlaender $=$ Theodor Fontane. Briefe an Georg Friedlaender, hg. und erläutert von Kurt Schreinert. Heidelberg 1954.

Briefe Hertz $=$ Theodor Fontane. Briefe an Wilhelm und Hans Hertz 1859. 1898 , hg. von Kurt Schreinert, vollendet und mit einer Einführung versehen von Gerhard Hay. Stuttgart 1972.

Fontane-Lepel $=$ Theodor Fontane und Bernhard von Lepel. Ein Freund schaftsbriefwechsel, hg. von Julius Petersen, 2 Bde. München 1940.

Propyläen Briefe 1-4 = Theodor Fontane. Briefe I-IV, hg. von Kurt Schreinert. $\mathrm{Zu}$ Ende geführt und mit einem Nachwort versehen von Charlotte Jolles. Berlin 1971-1975.

Tagebücher 1-2 = Theodor Fontane, Tagebücher 1852, 1855-1858, hg. von Charlotte Jolles unter Mitarbeit von Rudolf Muhs; Tagebücher 1866-1882, 1884-1898, hg. von Gotthart Erler unter Mitarbeit von Therese Erler. Berlin 1994.

Fontane-Chronik $=$ Christian Grawe, Fontane - Chronik. Stuttgart $1998(=\mathrm{Re}-$ clam UB).

Demetz $=$ Peter Demetz, Formen des Realismus: Theodor Fontane. Kritische Untersuchungen. Frankfurt/M u. a. 1973 (= Ullstein Tb. 2983).

Müller-Seidel $=$ Walter Müller-Seidel, Theodor Fontane. Soziale Romankunst in Deutschland. Stuttgart 1975.

Reuter = Hans-Heinrich Reuter, Fontane, 2 Bde. München 1968.

Wandrey = Conrad Wandrey, Theodor Fontane. München 1919.

Kleinschreibung am Anfang eines Zitats bedeutet, daß es sich nicht um einen Satzanfang handelt. Wo ein Zitat keine Angabe hat, gilt die nächstfolgende Angabe dafür mit.

Aus Platzgründen sind die Zahlen und bei den Zeitangaben die Monate nicht ausgeschrieben worden (Bsp. 20. 3. 1880 = 20. März 1880). 